\title{
Distribution of Mass and Energy in Closed Model of the Universe
}

\author{
Fadel A. Bukhari \\ Department of Astronomy, Faculty of Science, King Abdulaziz University, Jeddah, Saudi Arabia \\ Email: fdbukhari@gmail.com
}

Received 2 February 2015; accepted 21 December 2015; published 25 December 2015

Copyright (C) 2015 by author and Scientific Research Publishing Inc.

This work is licensed under the Creative Commons Attribution International License (CC BY). http://creativecommons.org/licenses/by/4.0/

(c) (i) Open Access

\section{Abstract}

The universe's horizon distance and volume are constructed in the closed cosmic model. The universe horizon distance distribution increases constantly for $t<t_{m e}$ and decreases for $t>t_{m e}$. However, the universe's horizon volume shows a sudden reduction in the range $t=0.5 \mathrm{Gyr}-t_{m e}$ due to the change of the universe space from flat to curved then closed in the interval $15.1261 \mathrm{Gyr} \leq t \leq$ $t_{m e}$. On the other hand, this distribution exhibits an abrupt rise in the range $t=t_{m e}-t_{*}$ due to the change of the universe space from closed then curved to flat in the interval $39.3822 \leq t \leq 40.7521$ Gyr. The mass of radiation, matter and dark energy within the horizon volume of the universe are also investigated. These distributions reveal similar noticeable changes as the universe's horizon volume distribution for the same reasons. The mass of radiation dominates up to $t=53221.5 \mathrm{yr}$, then the mass of matter becomes larger. Afterwards, both distributions of radiation and matter decrease while the distribution of dark energy rises until $t=10.1007 \mathrm{Gyr}$, where the mass of dark energy prevails up to $t=t_{\text {me }}$. Hence, the distribution of dark energy reduces until $t=40.2892 \mathrm{Gyr}$, where the mass of matter becomes prominent again. At $t=53.6246 \mathrm{Gyr}$ the masses of both matter and radiation become appreciably high such that the intercluster space will vanish and clusters of galaxies interfere with each other. Furthermore, not only the intergalactic medium will disappear, but also galaxies will collide and merge with each other to form extremely dense and close cosmological bodies. These very dense bodies will undergo further successive collisions and mergers under the action of central gravity, where the interstellar medium will vanish and the universe would develop to big crunch at $t_{b c}=53.6251 \mathrm{Gyr}$. It is interesting to note that the horizon distance of the universe in the closed model at $t=t_{m e}$ is in very good agreement with the maximum horizon distances in the five general cosmic models.

\section{Keywords}

Dark Energy, Radiation, Closed Cosmic Model 


\section{Introduction}

The distribution of density parameters of radiation, matter and dark energy in the closed cosmic model were investigated in a previous study [1], where we discovered the main epochs of the universe history in this model. It is worthy now to study the distributions of equivalent mass of radiation, mass of matter and equivalent mass of dark energy within the horizon volume of the universe to get deeper sight of the universe evolution in the closed model.

The reason for considering the equivalent mass of radiation in this study is the significant value of the radiation density parameter in the early universe and before the big crunch as we have seen in [1].

Therefore, it is vital to develop the distributions of the horizon distance and horizon volume of the universe in the closed model at various time ranges depending on the bases presented in [2]. Description of methodology is illustrated in Section 2, while algorithm would be shown in Section 3. Results and discussion are displayed in Section 4. Conclusion is given in Section 5.

\section{Methodology}

It is obvious from [2] that the horizon distance and horizon volume of the universe in closed cosmic model at the present time are respectively

$$
\begin{gathered}
d_{h}\left(t_{o}\right)=\frac{c}{H_{o}} \int_{0}^{1} \frac{1}{a}\left[1-S^{2} \Omega_{\Lambda, t}^{\prime \prime}\left(1-a^{2}\right)+S^{2} \Omega_{m, t}^{\prime \prime}\left(a^{2}-a^{3}\right)+S^{2} \Omega_{r, t}^{\prime \prime}\left(a^{2}-a^{4}\right)\right]^{-1 / 2} \mathrm{~d} a . \\
V_{h}\left(t_{o}\right)=\frac{8 \pi}{3} d_{h}^{3}\left(t_{o}\right) .
\end{gathered}
$$

where $\Omega_{\Lambda, t}^{\prime \prime}, \Omega_{m, t}^{\prime \prime}$ and $\Omega_{r, t}^{\prime \prime}$ are given by

$$
\begin{gathered}
\Omega_{\Lambda, t}^{\prime \prime}=\frac{\rho_{\Lambda, t}^{\prime}}{c^{2} \rho_{c, t}^{\prime \prime}} . \\
\Omega_{m, t}^{\prime \prime}=\frac{\rho_{m, t}^{\prime}}{\rho_{c, t}^{\prime \prime}} . \\
\Omega_{r, t}^{\prime \prime}=\frac{\rho_{r, t}^{\prime}}{c^{2} \rho_{c, t}^{\prime \prime}} . \\
\rho_{c, t}^{\prime \prime}=\frac{3 H^{\prime 2}(t)}{8 \pi G} . \\
\text { where } s=\frac{H(t)}{H_{o}}, S=\frac{H^{\prime}(t)}{H_{o}} \frac{H_{o}}{a}\left[1-s^{2} \Omega_{\Lambda, t}^{\prime}\left(1-a^{2}\right)+s^{2} \Omega_{m, t}^{\prime}\left(a^{2}-a^{3}\right)+s^{2} \Omega_{r, t}^{\prime}\left(a^{2}-a^{4}\right)\right]^{\frac{1}{2}} . \\
H(t)=\frac{H_{o}}{a}\left[1-\Omega_{\Lambda, o}\left(1-a^{2}\right)+\Omega_{m, o}\left(\frac{1}{a}-1\right)+\Omega_{r, o}\left(\frac{1}{a^{2}}-1\right)\right]^{\frac{1}{2}} . \\
\Omega_{\Lambda, t}^{\prime}=\frac{\rho_{\Lambda, t}^{\prime}}{c^{2} \rho_{c, t}} . \\
\Omega_{m, t}^{\prime}=\frac{\rho_{m, t}^{\prime}}{\rho_{c, t}} . \\
\Omega_{r, t}^{\prime}=\frac{\rho_{r, t}^{\prime}}{c^{2} \rho_{c, t}} .
\end{gathered}
$$




$$
\begin{gathered}
\frac{\rho_{\Lambda, t}^{\prime}}{c^{2}}=\rho_{c, o} \Omega_{\Lambda, o}-\Delta\left(\frac{\rho_{\Lambda, t}}{c^{2}}\right) . \\
\rho_{m, t}^{\prime}=\rho_{c, o} \frac{\Omega_{m, o}}{a^{3}}+\frac{1}{2} \Delta\left(\frac{\rho_{\Lambda, t}}{c^{2}}\right) . \\
\frac{\rho_{r, t}^{\prime}}{c^{2}}=\rho_{c, o} \frac{\Omega_{r, o}}{a^{4}}+\frac{1}{2} \Delta\left(\frac{\rho_{\Lambda, t}}{c^{2}}\right) . \\
\Delta\left(\frac{\rho_{\Lambda, t}}{c^{2}}\right)=0.01 \frac{\rho_{\Lambda, t}}{c^{2}} t .
\end{gathered}
$$

where $t$ is the cosmic time in Gyr.

$$
\rho_{c, t}=\frac{3 H^{2}}{8 \pi G} .
$$

The horizon distance of the universe in the closed cosmic model at any given time is given by

$$
d_{h}(t)=\frac{c}{H_{o}} \int_{0}^{a} \frac{1}{a}\left[1-S^{2} \Omega_{\Lambda, t}^{\prime \prime}\left(1-a^{2}\right)+S^{2} \Omega_{m, t}^{\prime \prime}\left(a^{2}-a^{3}\right)+S^{2} \Omega_{r, t}^{\prime \prime}\left(a^{2}-a^{4}\right)\right]^{-1 / 2} \mathrm{~d} a .
$$

Consequently, the change in the horizon distance of the universe in the time interval between two instants of scale factors $a_{1}, a_{2}$ is written as

$$
\Delta d_{h}(t)=\frac{c}{H_{o}} \int_{a_{1}}^{a_{2}} \frac{1}{a}\left[1-S^{2} \Omega_{\Lambda, t}^{\prime \prime}\left(1-a^{2}\right)+S^{2} \Omega_{m, t}^{\prime \prime}\left(a^{2}-a^{3}\right)+S^{2} \Omega_{r, t}^{\prime \prime}\left(a^{2}-a^{4}\right)\right]^{-1 / 2} \mathrm{~d} a .
$$

The horizon volume of the universe in the closed model at any given time is expressed as

$$
V_{h}(t)=f\left(d_{h}(t), k\right) \text {. }
$$

Equation (18) indicates that the horizon volume of the universe at $t$ is a function of $d_{h}(t)$ and the curvature of space $k$ at $t$. Since this curvature could be flat, open and closed from the big bang to big crunch as evident from Table 3 in [1]. Thus, the low of $V_{h}(t)$ can be determined according to the value of $k$ at $t$, as explained in the following cases:

(1) Flat space $(k=0)$

We have seen in [2] that the horizon volume of the universe at time $t$ in this case is given by

$$
V_{h}(t)=\frac{8 \pi}{3} d_{h}^{3}(t) .
$$

Therefore, it is obvious from Table 3 in [1] that Equation (19) is used in the time intervals $t \leq 6.5321 \mathrm{Gyr}$, $10.0751<t \leq 15.1261 \mathrm{Gyr}, 39.3822<t \leq 40.7521 \mathrm{Gyr}$, and $53.48<t \leq t_{b c}$.

(2) Closed space $(k=+1)$

We recall the equation of proper distance of extragalactic object

$$
d_{p}(t)=R(t) f\left(r_{o}\right) .
$$

where

$$
f\left(r_{o}\right)=\int_{0}^{r_{o}} \frac{\mathrm{d} r}{\sqrt{1-k r^{2}}}= \begin{cases}\sin ^{-1} r_{o} & k=+1 \\ r_{o} & k=0 \\ \sinh ^{-1} r_{o} & k=-1\end{cases}
$$

And the volume of space within $d_{p}(t)$ is expressed as

$$
V_{p}(t)=2 R^{3}(t) \int_{0}^{2 \pi} \mathrm{d} \phi \int_{0}^{\pi} \sin \theta \mathrm{d} \theta \int_{0}^{r_{o}} \frac{r^{2}}{\sqrt{1-k r^{2}}} \mathrm{~d} r .
$$


where $R(t), r_{o}, r, \theta$ and $\phi$ are defined as in [3] [4]. For $k=+1$, Equations (21), (20) and (22) yield

$$
d_{p}(t)=R(t) \sin ^{-1} r_{o}
$$

Hence Equation (22) gives

$$
V_{p}(t)=8 \pi R^{3}(t) \int_{0}^{r_{0}} \frac{r^{2} d r}{\sqrt{1-r^{2}}}
$$

Assume

$$
r=\sin \alpha, \mathrm{d} r=\cos \alpha \mathrm{d} \alpha, \cos \alpha=\sqrt{1-r^{2}}
$$

Substituting by (25) in (24) we get

$$
V_{p}(t)=4 \pi R^{3}(t) \int_{0}^{\sin ^{-1} r_{o}}(1-\cos 2 \alpha) \mathrm{d} \alpha
$$

Let

$$
\beta=2 \alpha, \mathrm{d} \beta=2 \mathrm{~d} \alpha .
$$

Substituting by (27) in (26) we have

$$
V_{p}(t)=4 \pi R^{3}(t)\left(\sin ^{-1} r_{o}\right)^{3}\left[\frac{1}{\left(\sin ^{-1} r_{o}\right)^{2}}-\frac{r_{o} \sqrt{1-r_{o}^{2}}}{\left(\sin ^{-1} r_{o}\right)^{3}}\right] .
$$

Substituting by (23) in (28) yields

$$
V_{p}(t)=4 \pi d_{p}^{3}(t)\left[\frac{1}{\left(\sin ^{-1} r_{o}\right)^{2}}-\frac{r_{o} \sqrt{1-r_{o}^{2}}}{\left(\sin ^{-1} r_{o}\right)^{3}}\right] .
$$

Suppose $r_{o}=1$, hence $\alpha_{o}=\frac{\pi}{2}$ and Equation (29) becomes

$$
V_{p}(t)=\frac{16}{\pi} d_{p}^{3}(t)
$$

Thus, the horizon volume of the universe in the closed cosmic model at time $t$ in this case is expressed as

$$
V_{h}(t)=\frac{16}{\pi} d_{h}^{3}(t) .
$$

It is evident form Table 3 in [1] that Equation (23) is used in two time intervals extending through $15.1261<t \leq 39.3822$ Gyr.

(3) Open space $(k=-1)$

Equations (21), (20) and (22) give

$$
\begin{gathered}
d_{p}(t)=R(t) \sinh ^{-1} r_{o} \\
V_{p}(t)=8 \pi R^{3}(t) \int_{0}^{r_{0}} \frac{r^{2} \mathrm{~d} r}{\sqrt{1+r^{2}}} .
\end{gathered}
$$

Assume

$$
r=\sinh \xi, \mathrm{d} r=\cosh \xi \mathrm{d} \xi, \cosh \xi=\sqrt{1+r^{2}}
$$

Substituting by (34) in (33) we have

$$
V_{p}(t)=4 \pi R^{3}(t) \int_{0}^{\sinh ^{-1} r_{o}}(\cosh 2 \xi-1) \mathrm{d} \xi .
$$


Let

$$
\eta=2 \xi, \mathrm{d} \eta=2 \mathrm{~d} \xi
$$

Substituting by (36) in (35) we get

$$
V_{p}(t)=4 \pi R^{3}(t)\left(\sinh ^{-1} r_{o}\right)^{3}\left[\frac{r_{o} \cosh \left(\sinh ^{-1} r_{o}\right)}{\left(\sinh ^{-1} r_{o}\right)^{3}}-\frac{1}{\left(\sinh ^{-1} r_{o}\right)^{2}}\right] .
$$

Substituting by (32) in (37) yields

$$
V_{p}(t)=4 \pi d_{p}^{3}(t)\left[\frac{r_{o} \cosh \left(\sinh ^{-1} r_{o}\right)}{\left(\sinh ^{-1} r_{o}\right)^{3}}-\frac{1}{\left(\sinh ^{-1} r_{o}\right)^{2}}\right] .
$$

When $r_{o}=1$, Equation (38) reduces to

$$
V_{p}(t)=3.112983 \pi d_{p}^{3}(t) .
$$

Therefore, the horizon volume of the universe in closed cosmic model at time $t$ in this case is written as

$$
V_{h}(t)=3.112983 \pi d_{h}^{3}(t) .
$$

It is clear form Table 3 in [1] that Equation (40) is used in the time intervals $6.5321<t \leq 10.0751 \mathrm{Gyr}$, $40.7521<t \leq 53.48 \mathrm{Gyr}$.

The total density of the universe in the closed cosmic model at time $t$ is

$$
\rho^{\prime}(t)=\rho_{m, t}^{\prime}+\frac{\rho_{r, t}^{\prime}}{c^{2}}+\frac{\rho_{\Lambda, t}^{\prime}}{c^{2}}
$$

Substituting by (3)-(5) in (41) we get

$$
\begin{gathered}
\rho^{\prime}(t)=\rho_{c, t}^{\prime \prime} \Omega_{m, t}^{\prime \prime}+\rho_{c, t}^{\prime \prime} \Omega_{r, t}^{\prime \prime}+\rho_{c, t}^{\prime \prime} \Omega_{\Lambda, t}^{\prime \prime} \\
\rho^{\prime}(t)=\rho_{c, t}^{\prime \prime} \Omega^{\prime \prime}(t) .
\end{gathered}
$$

where

$$
\Omega^{\prime \prime}(t)=\Omega_{m, t}^{\prime \prime}+\Omega_{r, t}^{\prime \prime}+\Omega_{\Lambda, t}^{\prime \prime} .
$$

From Equation (19), (30), (40) and (42) the total mass of the universe within the horizon volume in closed cosmic model at time $t$ is

$$
M_{h}(t)=V_{h}(t) \rho^{\prime}(t)
$$

The masses of matter, radiation and dark energy within the horizon volume of the universe in closed cosmic model at time $t$ are respectively

$$
\begin{gathered}
M_{m, t}=M_{h}(t) \frac{\Omega_{m, t}^{\prime \prime}}{\Omega^{\prime \prime}(t)} . \\
M_{r, t}=M_{h}(t) \frac{\Omega_{r, t}^{\prime \prime}}{\Omega^{\prime \prime}(t)} . \\
M_{\Lambda, t}=M_{h}(t) \frac{\Omega_{\Lambda, t}^{\prime \prime}}{\Omega^{\prime \prime}(t)} .
\end{gathered}
$$

The time interval between two instants with scale factors $a_{1}, a_{2}$ during the universe expansion is given by Equation (16) in [4] [5] as

$$
\Delta t=\frac{1}{H_{o}} \int_{a_{1}}^{a_{2}}\left[1-\Omega_{\Lambda, o}\left(1-a^{2}\right)+\Omega_{m, o}\left(\frac{1}{a}-1\right)+\Omega_{r, o}\left(\frac{1}{a^{2}}-1\right)\right]^{-\frac{1}{2}} \mathrm{~d} a .
$$


However, during the universe contraction if $a_{2}<a_{1}$ then modulus of the right hand side of (50) should be taken.

\section{Algorithm}

In determination of the distributions of $d_{h}(t), V_{h}(t), M_{h}(t), M_{m, t}, M_{r, t}$ and $M_{\Lambda, t}$ we use the following steps:

(1) Stage of the universe expansion.

a) Set $t=0, d_{h}=0$ and insert the value of $a_{\max }=0.093188, J=1000$ for $\mathrm{t} \leq 0.5 \mathrm{Gyr}, a_{\max }=2.3755873$, $J=1600$ for $0.5<t \leq t_{m e}$.

b) Calculate $D A=\frac{a_{\max }}{D B L E(J)}$.

c) Start general DO loop $I=2, J$ which includes the following sub steps:

d) $a_{1}=D A(I-1), a_{2}=D A I$.

e) Compute new value of cosmic time $t$ numerically using (48), where $t=t+\Delta t$.

f) Obtain new value of the universe horizon distance $d_{h}$ numerically using (17-b), where $d_{h}=d_{h}+\Delta d_{h}$.

g) Determine the corresponding values of $V_{h}$ using (19), (31) and (40), in addition to the values of $H^{\prime}(t), \rho_{c, t}^{\prime \prime}, \Omega_{m, t}^{\prime \prime}, \Omega_{r, t}^{\prime \prime}, \Omega_{\Lambda, t}^{\prime \prime}, \Omega^{\prime \prime}(t), \rho^{\prime}(t), M_{h}(t), M_{m, t}, M_{r, t}$ and $M_{\Lambda, t}$ using (7), (6), (4), (5), (3), (43), (42), (44), (45), (46) and (47) respectively.

h) Continue the general DO loop.

(2) Stage of the universe contraction

a) Set $t=t_{m e}=26.8125327 \mathrm{Gyr}, d_{h}=d_{h}\left(t_{m e}\right)=18.619224 \mathrm{Gpc}$, and insert the values of $a_{\max }=2.3755873, J 1=1600$ for $t_{\text {me }}<t \leq t_{*}, t_{*}=t_{b c}-0.5 \mathrm{Gyr}, a_{\max }=0.093539, J 1=90$ for $t>t_{*}$.

b) Evaluate $D A=\frac{a_{\max }}{D B L E(J 1)}$.

c) Start general DO loop $I=1, J 1$ which includes the following sub steps:

d) Set $J 2=J 1-I+1, a_{1}=D A(J 2-1), a_{2}=D A J 2$.

e) Obtain new value of cosmic time $t$ numerically using (48), where $t=t+\Delta t$.

f) Compute new value of the universe horizon distance $d_{h}$ numerically using (17-b), where $d_{h}=d_{h}-\Delta d_{h}$. Sub steps (g) and (h) are similar to (g), (h) mentioned above in the stage of the universe expansion.

\section{Results and Discussion}

The distribution of the universe horizon distance in the closed cosmic model until $t=0.5 \mathrm{Gyr}$ is shown in Figure 1(a). The distribution increases quite slowly up to $t=5.8780 \mathrm{Myr}$, then the distribution starts raising rapidly. However, the distribution of the universe horizon distance in the range $t=0.5 \mathrm{Gyr}-t_{m e}$ increases very fast until about $t=5.7237 \mathrm{Gyr}$. Afterwards, it raises gradually as indicated in Figure 1(b). Furthermore, the distribution of the universe horizon distance in the range $t=t_{m e}-t_{*}$, where $t_{*}=t_{b c}-0.5 \mathrm{Gyr}$, decreases quite slowly up to $t=46.5790 \mathrm{Gyr}$, hence it decreases relatively fast as presented in Figure 1(c). Nevertheless, the distribution of the universe horizon distance in the range $t=t_{*}-t_{n}$ decreases slowly until $t=53.4733 \mathrm{Gyr}$, $t_{n}=t_{b c}-0.0005 \mathrm{Gyr}$, then it starts reduction sharply towards $t=t_{b c}$ as displayed in Figure 1(d).

The distribution of the universe horizon volume in the closed cosmic model up to $t=0.5 \mathrm{Gyr}$ is shown in Figure 2(a). The distribution increases very slowly until $t=18.4785 \mathrm{Myr}$. Afterwards, the distribution starts raising appreciably. During this cosmic time range the space of universe is flat. The distribution of the universe horizon volume continues raising up to $t=14.8 \mathrm{Gyr}$, hence the distribution suddenly decreases until $t=16.6667 \mathrm{Gyr}$, then it increases gradually up to $t=t_{\text {me }}$ as seen is Figure 2(b). The sharp decrease of the distribution in the range $14.8<t<16.6667 \mathrm{Gyr}$ because the space of the universe changes from flat to curved then closed in the interval $15.1261 \mathrm{Gyr} \leq t \leq t_{m e}$.

The distribution of the universe horizon volume in the range $t=t_{m e}-t_{*}$ is disclosed in Figure 2(c). The distribution decreases until $t=37.6974 \mathrm{Gyr}$, hence it shows abrupt raising up to $t=40.6579 \mathrm{Gyr}$, hence it reduces again until $t=t_{*}$. The abrupt increase of the distribution in the range $37.6974<t<40.6579 \mathrm{Gyr}$ is due to 


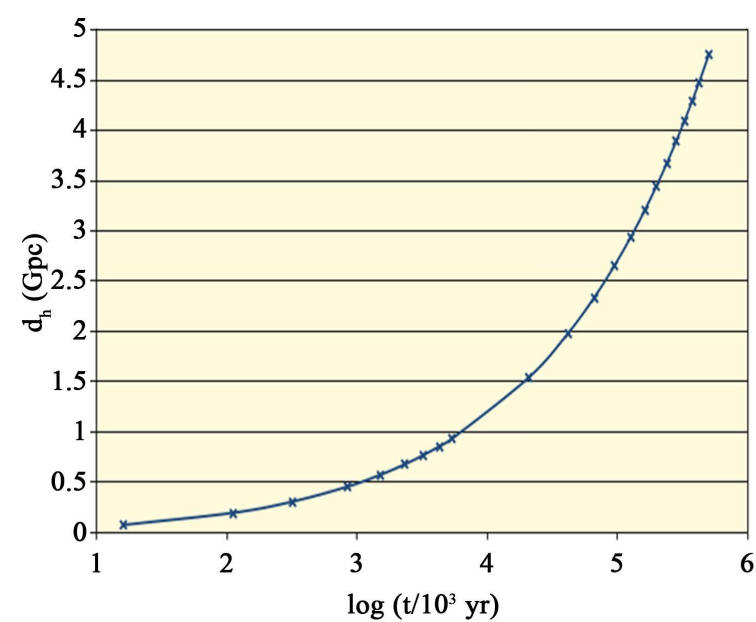

(a)

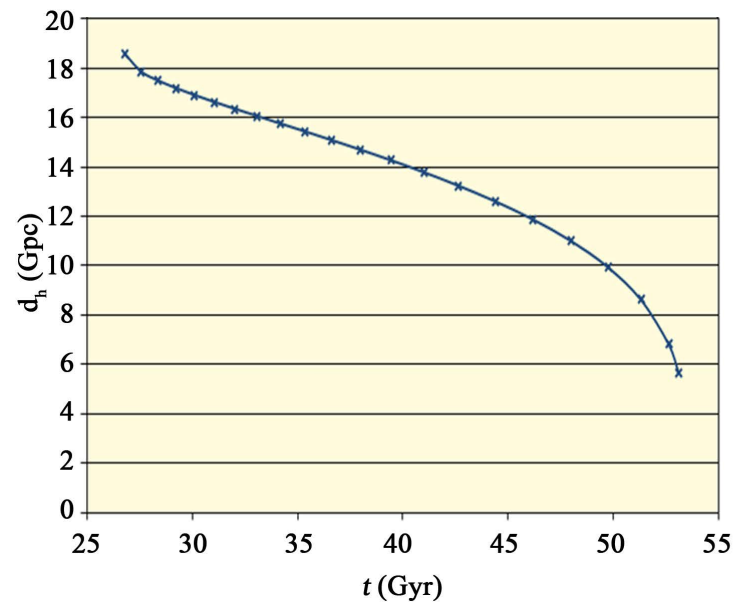

(c)

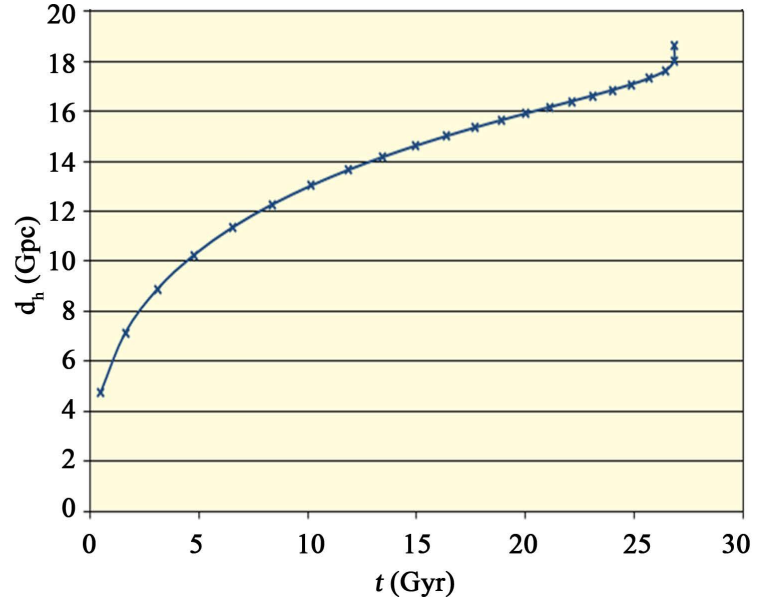

(b)

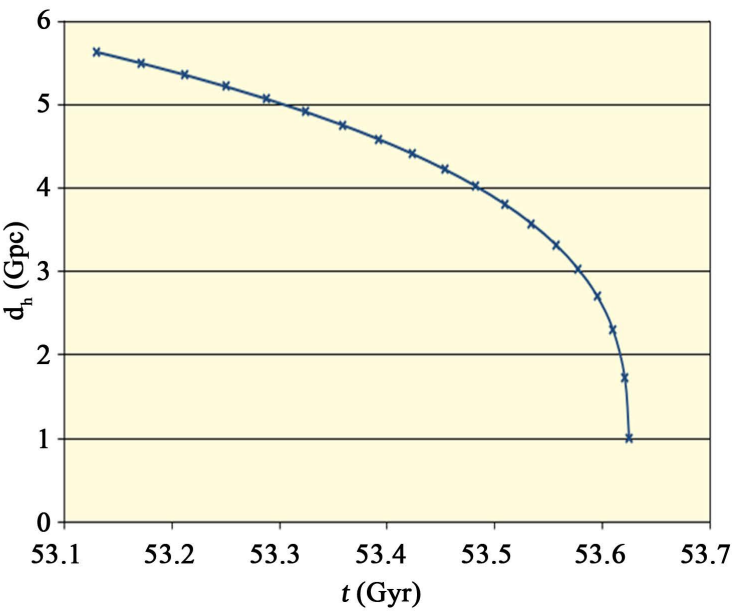

(d)

Figure 1. (a) The distribution of the universe horizon distance in the closed cosmic model up to $t=0.5$ Gyr; (b) The distribution of the universe horizon distance in the closed cosmic model in the range $t=0.5 \mathrm{Gyr}-t_{\mathrm{me}}$; (c) The distribution of the universe horizon distance in the closed cosmic model in the range $t=t_{\mathrm{me}}-t_{*}$; (d) The distribution of the universe horizon distance in the closed cosmic model in the range $t=t_{*}-t_{n}$.

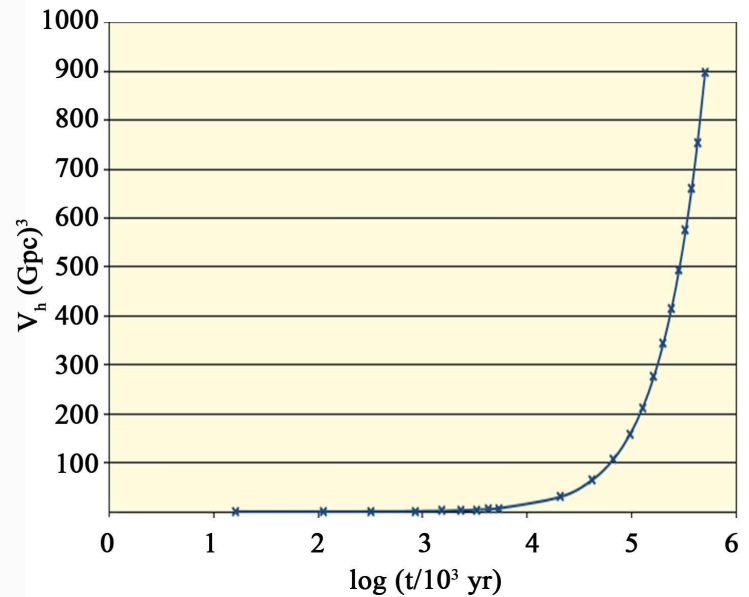

(a)

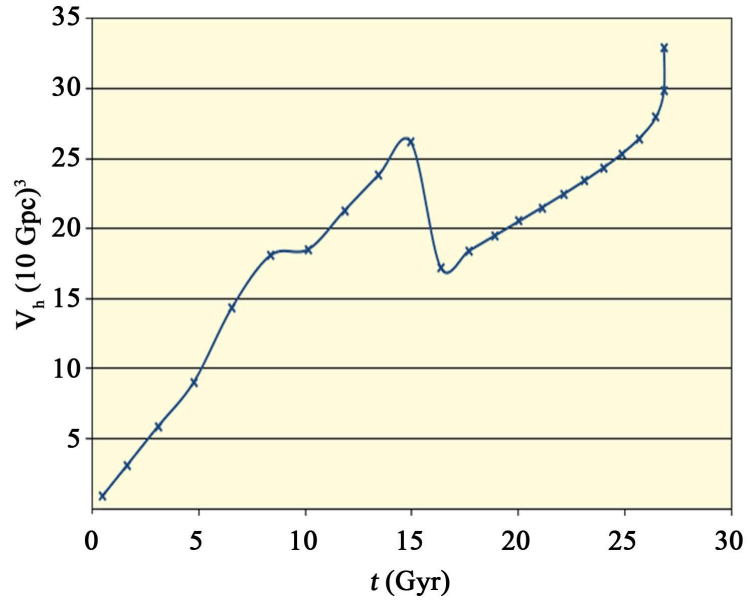

(b) 


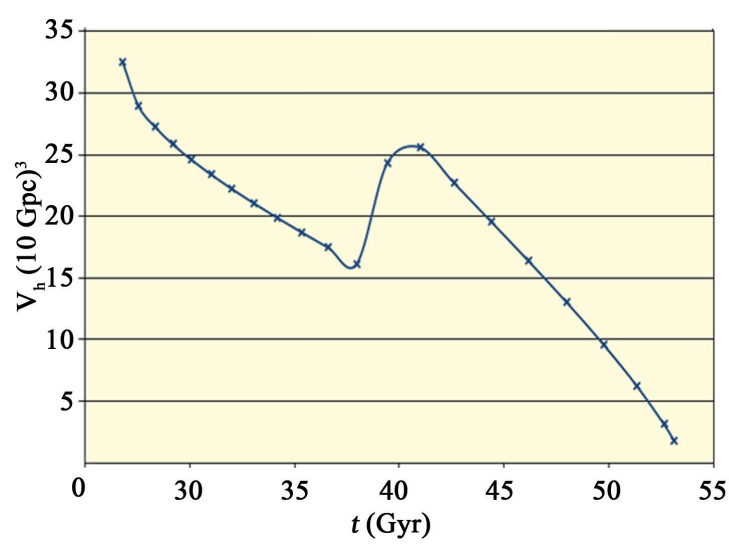

(c)

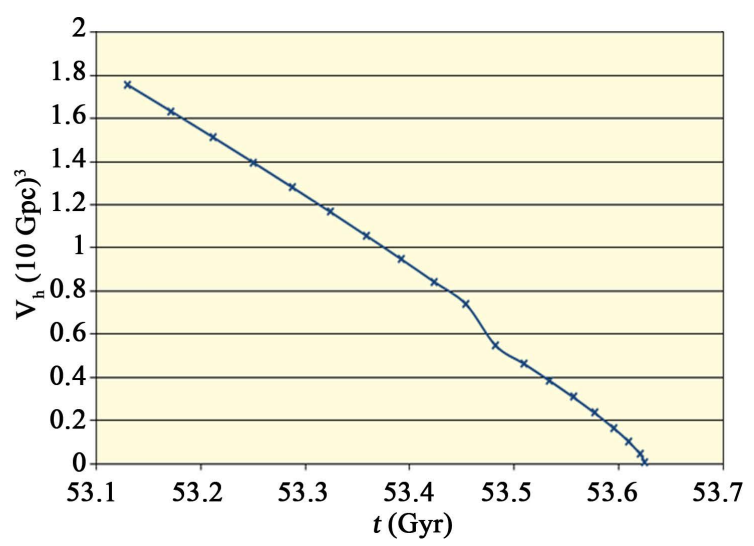

(d)

Figure 2. (a) The distribution of the universe horizon volume in the closed cosmic model up to $t=0.5$ Gyr; (b) The distribution of the universe horizon volume in the closed cosmic model in the range $t=0.5 \mathrm{Gyr}-t_{\mathrm{me}}$; (c) The distribution of the universe horizon volume in the closed cosmic model in the range $t=t_{\mathrm{me}}-t_{*}$; (d) The distribution of the universe horizon volume in the closed cosmic model in the range $t=t_{*}-t_{n}$.

the fact that the universe space changes from closed then curved to flat in the interval $39.3822 \leq t \leq 40.7521$ Gyr. The distribution of the universe horizon volume in the range $t=t_{*}-t_{n}$ is presented in Figure 2(d), which is reducing gradually towards $t=t_{b c}$.

The distribution of mass and energy within the horizon volume of the universe in the closed cosmic model until $t=0.5 \mathrm{Gyr}$ is shown in Figure 3(a). The distributions of radiation and total mass decrease very slowly up to $t=134596 \mathrm{yr}$ and $t=162061 \mathrm{yr}$ respectively, then they reduce more rapidly. However, the distribution of matter increases so slowly until $t=181161 \mathrm{yr}$ and it decreases rapidly afterwards. This distribution intersects with the distribution of radiation at $t=53221.5 \mathrm{yr}$ and coincides with the distribution of total mass at $t=3.2819 \mathrm{Myr}$. The distribution of dark energy increases continuously towards $t=0.5 \mathrm{Gyr}$. The distribution of mass and energy within the horizon volume of the universe in the closed model in the range $t=0.5 \mathrm{Gyr}-t_{m e}$ is illustrated in Figure 3(b). It is noticeable that the distribution of radiation intersects the distribution of dark energy at $t=0.6618 \mathrm{Gyr}$, hence it reduces very fast up to $t=3.0147 \mathrm{Gyr}$. Afterwards, it raises gradually until $t=14.9265 \mathrm{Gyr}$, then it decreases abruptly up to $t=16.3971 \mathrm{Gyr}$, where it starts increasing again towards $t_{m e}$. The distribution of matter decreases up to $t=22.2794 \mathrm{Gyr}$, hence it raises very slowly towards $t_{m e}$. This distribution coincides with the distribution of total mass up to $t=1.9853 \mathrm{Gyr}$, and intersects with the dark energy distribution at $t=10.1007 \mathrm{Gyr}$. The matter distribution indicates obvious decrease in the range

$t=14.9265-16.3971 \mathrm{Gyr}$. The distribution of dark matter increases until $t=14.9265 \mathrm{Gyr}$, hence it also shows marked decrease up to $t=16.3971 \mathrm{Gyr}$. Afterwards, it raises slightly towards $t_{m e}$. The total mass distribution reduces up to $t=14.9265 \mathrm{Gyr}$, then it decreases abruptly until $t=16.3971 \mathrm{Gyr}$. Hence, this distribution increases gradually towards $t_{\text {me }}$. The noticeable decrease of all four distributions in the range about $14.9225<t<16.3971 \mathrm{Gyr}$ is due to the change of the universe space from flat to curved then closed in the range $15.1261 \leq t \leq t_{m e}$.

The distribution of mass and energy within the horizon volume of the universe in the closed model in the range $t=t_{m e}-t_{*}$ is illustrated in Figure 3(c). The distribution of radiation reduces very slowly until $t=37.9839 \mathrm{Gyr}$, then it raises suddenly up to $t=40.4032 \mathrm{Gyr}$. Hence, this distribution decreases again until $t=52.5807 \mathrm{Gyr}$ where it intersects with the distribution of dark energy, then it starts raising towards $t_{*}$. The matter distribution increases so slowly up to $t=37.9839 \mathrm{Gyr}$, hence it exhibits marked raise until $t=40.4032 \mathrm{Gyr}$ and intersects with the dark energy distribution at $t=40.2892 \mathrm{Gyr}$. Afterwards, the distribution of matter shows substantial increase towards $t_{*}$ and coincides with the total mass distribution at $t=51.2903 \mathrm{Gyr}$ hence forth. The dark energy distribution reduces gradually until $t=37.9839 \mathrm{Gyr}$ where it displays outstanding raise up to $t=40.4032 \mathrm{Gyr}$, hence it decreases again towards $t_{*}$. The total mass distribution reduces also gradually until $t=37.9839 \mathrm{Gyr}$, then it exhibits obvious increase up to $t=40.4032 \mathrm{Gyr}$. Afterwards, it raises very fast as the matter distribution. The prominent increase of the four distributions in the interval about $37.9839<t<40.4032 \mathrm{Gyr}$ is owing to the change of the universe space from closed then curved 


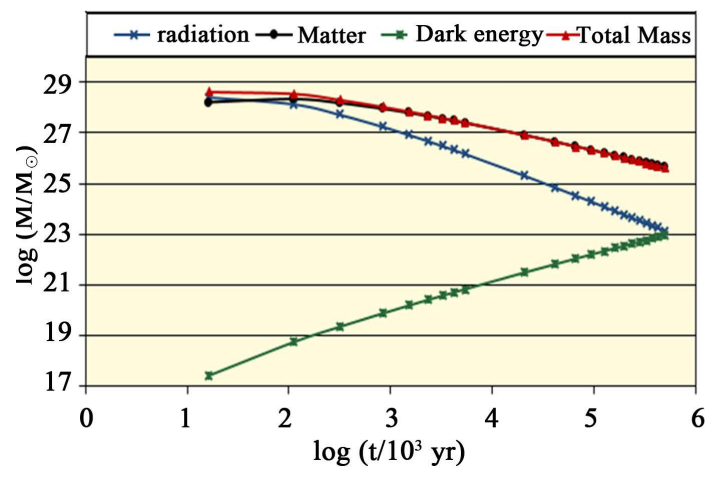

(a)

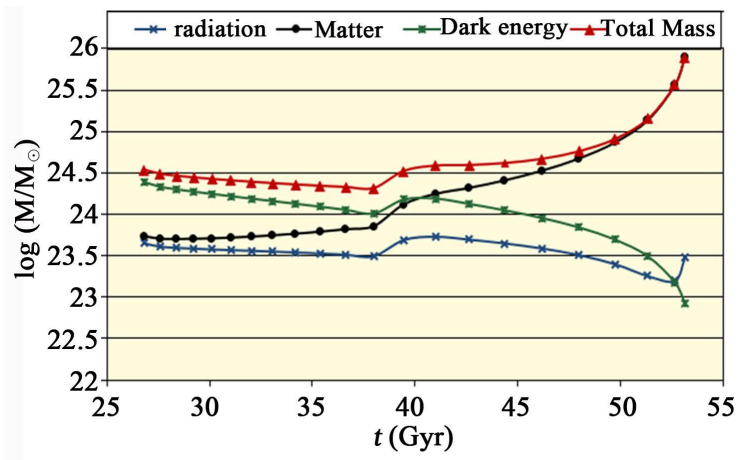

(c)

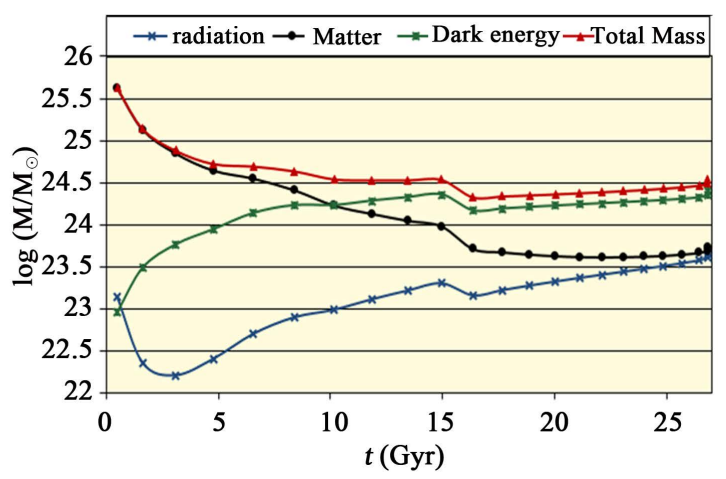

(b)

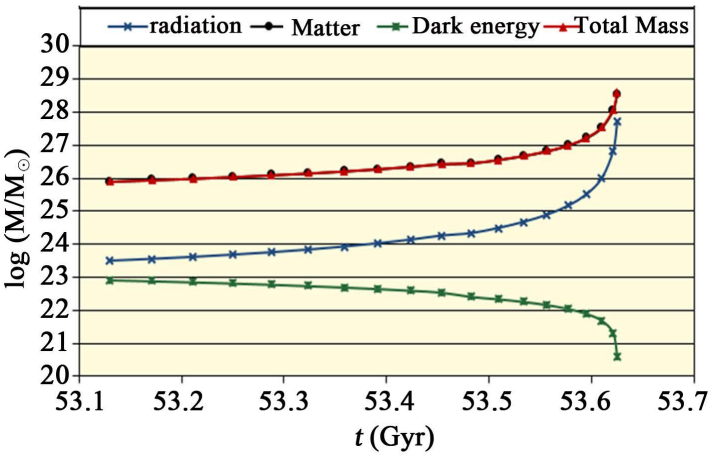

(d)

Figure 3. (a) The distribution of mass and energy in the closed cosmic model up to $t=0.5$ Gyr; (b) The distribution of mass and energy in the closed cosmic model in the range $t=0.5 \mathrm{Gyr}-t_{\mathrm{me}}$; (c) The distribution of mass and energy in the closed cosmic model in the range $t=t_{\mathrm{me}}-t_{*}$; (d) The distribution of the mass and energy in the closed cosmic model in the range $t=$ $t_{*}-t_{n}$.

to flat in the range $39.3822 \leq t \leq 40.7521 \mathrm{Gyr}$. The distribution of mass and energy within the horizon volume of the universe in the closed model in the range $t=t_{*}-t_{n}$ is displayed in Figure 3(d). The radiation distribution increases quite slowly until $t=53.5032 \mathrm{Gyr}$, hence it starts raising appreciably fast.

The distribution of both matter and total mass coincide on each other and lie over the radiation distribution. The two distributions increase gradually up to $t=53.5274 \mathrm{Gyr}$, then they raise up. However, the dark energy distribution decreases so slowly until $t=53.5742 \mathrm{Gyr}$, afterwards it reduces substantially.

Estimations of $d_{h}(t), V_{h}(t), M_{r}(t), M_{m}(t), M_{\wedge}(t)$ and the equivalent number of the Coma-like clusters to the mass of matter within the universe horizon volume $N_{\text {СомA }}(t)$ in the closed cosmic model at special times are presented in Table 1. It is interesting to note that at $t=t_{n}=t_{b c}-0.0005 \mathrm{Gyr}$, the horizon volume of the universe $V_{h}\left(t_{n}\right)=0.0003(10 \mathrm{Gpc})^{3}$.

Since the radius of the Coma cluster is $r_{\text {СOMA }}=3.6 \mathrm{Mpc}$ [5], then $V_{h}\left(t_{n}\right)=1.5738 \times 10^{6} V_{\text {COMA }}$, where $V_{\text {COMA }}$ is the Coma cluster volume. However, the mass of matter within the horizon volume of the universe at $t=t_{n}$ is $M_{m}\left(t_{n}\right)=1.7455 \times 10^{13} M_{\text {СОMA }}$. This indicates very clearly that the intercluster medium will disappear at $t=t_{n}$ and galaxy clusters will interfere with each other. Furthermore, the radius of the Milky Way galaxy is

$r_{M w}=50 \mathrm{Kpc}$ [6]. Thus, $V_{h}\left(t_{n}\right)=5.8742 \times 10^{11} V_{M W}$, where $V_{M W}$ is the Milky Way galaxy volume. Nevertheless, it is found that $M_{m}\left(t_{n}\right)=4.3638 \times 10^{16} M_{M W}$. Therefore, not only the intergalactic spaces will vanish at $t=t_{n}$, but also galaxies will collide and merge with each other to form extremely dense and close cosmological bodies. These very dense bodies will undergo further successive collisions and mergers under the action of central gravity, where the interstellar medium will vanish and the universe would develop to big crunch at $t_{b c}=53.6251 \mathrm{Gyr}$.

It is also interesting to note from Table 1 that the horizon distance of the universe at maximum expansion is $d_{h}\left(t_{m e}\right)=18.6192 \mathrm{Gpc}$. This horizon distance is in very good agreement with the maximum value of the universe horizon distance in the observed general cosmic model A, $d_{h}\left(t_{n}^{\prime}\right)=19.0103 \mathrm{Gpc}$, where $t_{n}^{\prime}=124 \mathrm{Gyr}$. 
Table 1. Estimations of the horizon distance, horizon volume, mass of radiation, mass of matter, mass of dark energy and the equivalent number of the Coma-like clusters to the mass of matter within the universe horizon volume in the closed cosmic model at special times.

\begin{tabular}{|c|c|c|c|c|c|c|}
\hline$t$ & $d_{h}(t)$ Gpc & $V_{h}(t)$ & $\log \left(M_{r} / M_{\odot}\right)$ & $\log \left(M_{m} / M_{\odot}\right)$ & $\log \left(M_{\wedge} / M_{\odot}\right)$ & $N_{\text {СомА }}(t)$ \\
\hline $\begin{array}{c}t_{r m} \\
(53221.5) \mathrm{yr}\end{array}$ & 0.1356 & $0.0209 u_{1}$ & 28.4321 & 28.4321 & 18.3292 & $1.352 u_{3}$ \\
\hline $\begin{array}{c}t_{m \wedge 1} \\
(10.1007) \mathrm{Gyr}\end{array}$ & 12.9717 & $18.2854 u_{2}$ & 22.9777 & 24.2252 & 24.2252 & $8.3970 u_{4}$ \\
\hline $\begin{array}{c}t_{o} \\
(13.7) \mathrm{Gyr}\end{array}$ & 14.4420 & $25.2348 u_{2}$ & 23.2298 & 24.0515 & 24.3294 & $5.6291 u_{4}$ \\
\hline $\begin{array}{c}t_{m e} \\
(26.812 .53) \mathrm{Gyr}\end{array}$ & 18.6192 & $32.8742 u_{2}$ & 23.6535 & 23.7347 & 24.3906 & $2.7141 u_{4}$ \\
\hline $\begin{array}{c}t_{m \wedge 2} \\
(40.2892) \mathrm{Gyr}\end{array}$ & 14.0058 & $23.0168 u_{2}$ & 23.6758 & 24.1474 & 24.1474 & $7.0198 u_{4}$ \\
\hline $\begin{array}{c}t_{n} \\
(53.6246) \mathrm{Gyr}\end{array}$ & 0.9972 & $0.0003 u_{2}$ & 27.7134 & 28.5430 & 20.5951 & $1.7455 u_{3}$ \\
\hline
\end{tabular}

where $t_{n}=t_{b c}-0.0005 \mathrm{Gyr}, u_{1}=(\mathrm{Gpc})^{3}, u_{2}=(10 \mathrm{Gpc})^{3}, u_{3}=10^{13}$ and $u_{4}=10^{8}$.

The value of $d_{h}\left(t_{m e}\right)$ is also in high agreement with the values of $d_{h}\left(t_{n}^{\prime}\right)$ in the other four general cosmic models as shown from Table 1 in [7].

\section{Conclusions}

In this paper we have investigated the distributions of the universe horizon distance and the universe horizon volume in the closed cosmic model. It is found that the universe horizon distance distribution increases constantly for $t<t_{m e}$ and decreases for $t>t_{m e}$. However, the universe horizon volume distribution shows sudden reduction in the range $t=0.5 \mathrm{Gyr}-t_{m e}$ due to the change of the universe space from flat to curved then closed in the interval $15.1261 \mathrm{Gyr} \leq t \leq t_{m e}$. On the other hand, this distribution exhibits abrupt raise in the range $t=t_{m e}-t_{*}$ because of the change of the universe space from closed then curved to flat in the interval $39.3822 \leq t \leq 40.7521 \mathrm{Gyr}$.

Distributions of mass of radiation, matter and dark energy within the horizon volume of the universe were also investigated in the closed cosmic model. These distributions reveal similar noticeable changes as the universe horizon volume distribution for the same reasons. The mass of radiation dominates up to $t=53221.5 \mathrm{yr}$, then the mass of matter becomes larger. Afterwards, both distributions of radiation and matter decrease while the distribution of dark energy rises until $t=10.1007 \mathrm{Gyr}$, where the mass of dark energy prevails up to $t=t_{m e}$. Hence, the distribution of dark energy reduces until $t=40.2892 \mathrm{Gyr}$ where the mass of matter becomes prominent again. At $t=53.6246 \mathrm{Gyr}$ the masses of both matter and radiation become appreciably high such that the intercluster space will vanish and clusters of galaxies will interfere with each other. Furthermore, not only the intergalactic medium will disappear, but also galaxies will collide and merge with each other to form extremely dense and close cosmological bodies. These very dense bodies will undergo further successive collisions and mergers under the action of central gravity, where the interstellar medium will vanish and the universe would develop to big crunch at $t_{b c}=53.6251 \mathrm{Gyr}$.

\section{References}

[1] Bukhari, F.A. (2013) A Closed Model of the Universe. International Journal of Astronomy and Astrophysics, 3, 189198. http://dx.doi.org/10.4236/ijaa.2013.32022

[2] Bukhari, F.A. (2013) Cosmological Distances in Closed Model of the Universe. International Journal of Astronomy and Astrophysics, 3, 199-203. http://dx.doi.org/10.4236/ijaa.2013.32023

[3] Bukhari, F.A. (2013) Cosmological Distances in Five General Cosmic Models. International Journal of Astronomy 
and Astrophysics, 3, 183-188. http://dx.doi.org/10.4236/ijaa.2013.32021

[4] Bukhari, F.A. (2013) Five General Cosmic Models. Journal of King Abdulaziz University: Science, 25.

[5] Ryden, B. (2003) Introduction to Cosmology. Addison \& Wesley, Boston.

[6] Schneider, P. (2010) Extragalactic Astronomy and Cosmology. Springer, New York.

[7] Bukhari, F.A. (2015) Distribution of Mass and Energy in Five General Cosmic Models. International Journal of Astronomy and Astrophysics, 5, 20-27. http://dx.doi.org/10.4236/ijaa.2015.51004 